\title{
Storm based Real-time Analytics Service on Propaganda and Sentiment Analysis of Political Tweets on Cloud Computing Environment
}

\author{
Akhmedov Khumoyun, Myoungjin Kim, Yun Cui, and Hanku Lee
}

\begin{abstract}
As mainstream social media and political campaigns begin to pay attention to the online political discourse, a taxonomic analysis of political speech in social media networks, such as Twitter, is becoming more crucial. What exactly people say on these sites, and how useful this data is in estimating political popularity could be very valuable source for Big Data Analytics. In this paper, we present design of Storm based Real-time Analytics Service (SRTAS) which is intended to extract tweets related to political context from public tweets and analyze their propaganda and sentiment rate using Storm and other Big Data systems such as Kafka. Propaganda can be effectively utilized to boost company's business revenues and enhance its reputation. Hence, in this work, propaganda and sentiment rate of political tweets leveraged to achieve the goal of the work, namely, finding political trend among mess of tweets in particular and the impact of political tweets on people on the whole.
\end{abstract}

Keywords — Real-time Analytics, Big Data, Storm, Twitter.

\section{INTRODUCTION}

$\mathrm{T}$ HE wide spread of World Wide Web has brought a new way of expressing opinions and feelings of individuals. It is also a medium with a vast amount of diverse information where users can view the opinion of other users that are classified into different groups, such as politics, health care, sports, fashion and so on. And these views might shape their attitude towards some particular subject and play a key factor in decision making. Therefore, enterprises, organizations, governments and different groups in general have shown their interest in the opinions that users have for their activities. They are also interested to the way how users facilitate these media, the communication behavior, and some user attributes such as age, political orientation, social interactions, etc. There has been considerable amount of work in the area of Sentiment Analysis and Opinion Mining with different, sometimes similar, approaches in order to analyze public standpoint of particular topic and find patterns and correlations among peoples' views and their impact on social media, and in turn on individuals too.

Humoyun Ahmedov is with Department of Internet \& Multimedia Engineering, Konkuk University, Seoul 143-701, South Korea.

Myoungjin Hyong was with Konkuk University, $\mathrm{He}$ is now with Innogrid Cloud Provider, Seoul, South Korea.

Yun Cui is with Department of Internet \& Multimedia Engineering Department, Konkuk University, Seoul, South Korea.

Hanku Lee is a professor at Division of Internet \& Multimedia Engineering, Konkuk University, Seoul, South Korea.
The approach which we have taken for this paper is somewhat different from previous related works as it attempted to combine propaganda rate with sentiment analysis of public tweets on political context in order to sufficiently illustrate social impact of political tweets on individuals. By propaganda rate, we meant that the role and frequency of domain specific words in political context, how they appear in a tweet and how it reflects the author's intention. Our work also heavily depends on Sentiment Analysis techniques and well-known Machine Learning algorithms such as Naïve Bayesian and Support Vector Machine (SVM). A wide set of techniques of Sentiment Analysis (SA) are used in micro-blogging texts to extract the polarity (positive, negative, neutral or mixed) that users express in these texts. In this regard, Twitter has become a famous micro-blogging site in which users express their opinions in real time. The texts in Twitter are called tweets, which can only contain maximum of 140 characters and a language that does not have any restriction on the form and content.

The paper is organized as follows. In section 2 we present previous relevant works for Twitter Sentiment Analysis on political context. In section 3 we give general information on Storm, Kafka and present Sentiment Analysis techniques and ML algorithms which we used in this work and also description of the corpus which we obtained so far, from 30.10.2014 till 30.08.2015, by Twitter Streaming API. Then, in section 4 we present the overall architecture, dataflow and workflow of the proposed system, Storm based Real Time Analytics Service (SRTAS). Finally, in the last section we present conclusions and possible directions for future work.

\section{RELATED WORK}

The recent role of social media in political actions in US, Europe, and elsewhere around the world has triggered a plenty of studies on mining of political speech online. For example, Y. R. Lin, J. P. Bagrow, [1] used social media to determine whether news sources are biased in favour of covering one political party more than another). There has been a lot of works in the area of Sentiment Analysis. Bo Pang and Lee were pioneers in this area. Current works in this area includes using a mathematical approach which uses a formula for the sentiment value depending on the proximity of the words with adjectives like 'excellent', 'worse', 'bad' etc. Besides, they used machine learning techniques to analyze product reviews [2], social media-driven sentiment analysis (SA) has recently expanded its 
target to encompass political discourse. Facilitating data mining and NLP tools, the aim is to determine the author's political opinion [3], diverging from the standard practice of focusing on sentiment defined as positive or negative.

Further, O'Connor et al.[4] figured out that surveys of consumer confidence and political opinion correlate with sentiment word frequencies in tweets, and offered text stream mining as a replacement for traditional polling. Jansen et al.[5] discuss the implications for organizations of using micro-blogging as part of their marketing strategy. Pak et al. [6] used classification based on the multinomial Na'ive Bayes classifier for sentiment analysis. Go et al.[7] compared multinomial Na'ıve Bayes, a maximum entropy classifier, and a linear support vector machine; they all exhibited broadly comparable accuracy on their test data, but small differences could be observed depending on the features used. Considering sentiment analysis on data streams, [8] was the first work that discussed the challenges that Twitter streaming data poses, and proposed the sliding window Kappa statistic as an evaluation metric for data streams. Considering all tests performed and ease of interpretability, this work recommended the SGD-based model, used with an appropriate learning rate, for the Twitter data stream. An advantage of the SGD-based model is that changes in its weights can be inspected to gain insight into changing properties of the data stream. Monitoring these coefficients, which determine how strongly absence/presence of the corresponding word influences the model's prediction of negative or positive sentiment, is also an efficient way to detect changes in the population's opinion.

As a last point, Barack Obama's 2008 Presidential campaign, the world saw the crowning of the Internet as the king of all political media" [9]. Online activity indicators such as number of fans on Facebook, followers on Twitter, and likes on YouTube have been seen as indicators of a galvanized base, which ultimately contributed to Obama's victory [10]. Since then, not only has traditional media started paying more attention to political discussions on social media, but several research papers have been published claiming a connection between social media and public polls and even election outcomes.

\section{TECHNOLOGIES AND TECHNIQUES}

Twitter might be described as a real-time, highly social microblogging service that allows users to post short status updates, called tweets, that appear on timelines. Tweets may include one or more entities in their 140 characters of content and reference one or more places that map to locations in the real world. An understanding of users, tweets, and timelines is particularly essential to effective use of Twitter's API, so a brief introduction to these fundamental Twitter Platform objects is in order before we interact with the API to fetch some data. A tweet is not only a simple text message but it is a combination of text data and Meta data associated with the tweet. These attributes are the features of tweets. They expresses the content of the tweet or what is that tweet about. The Metadata can be utilized to find out the domain of the tweet.

\section{A. Twitter Features}

Mention: in a tweet when user want to refer to another user he can write his name starting with@ symbol. It is called as Mention and it also represented as “@username”.

Hashtag: it is a word starting with \# symbol. It refers to a word about the content of text or indicating the topic of tweet.

Emoticons: these are facial expressions pictorially characterized using punctuation and letters; they express the user's mood.

Retweet: a tweet can be just a statement made by a user, or could be a reply to another tweet. Retweets are marked with either "RT" followed by ‘@user id' or "via @user id"

The nature of tweets introduces new challenges for researchers in Natural Language Processing (NLP). In some cases, the tweets are written with ungrammatical sentences with a lot of emoticons, abbreviations, specific terminology, slang, etc. Hence, the usual techniques of NLP must be adapted to these characteristics of the language, and new approaches must be proposed in order to successfully address this problem.

\section{B. Apache Storm}

Apache Storm is a free and open source distributed real-time computation system [14]. Storm makes it easy to reliably process unbounded streams of data, doing for real-time processing what Hadoop did for batch processing. Storm has simple API, can be used with any programming language, and is a lot of fun to use! Storm has many use cases: real-time analytics, online machine learning, continuous computation, distributed RPC, ETL, and more. Storm is fast: a benchmark clocked it at over a million tuples processed per second per node. It is scalable, fault-tolerant, guarantees your data will be processed, and is easy to set up and operate.

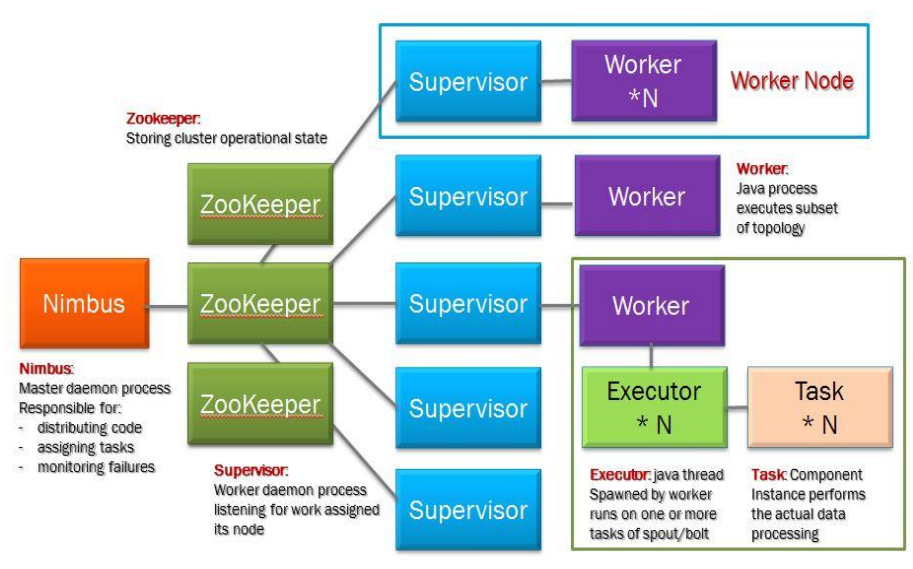

Fig. 1 Physical view of Storm cluster 


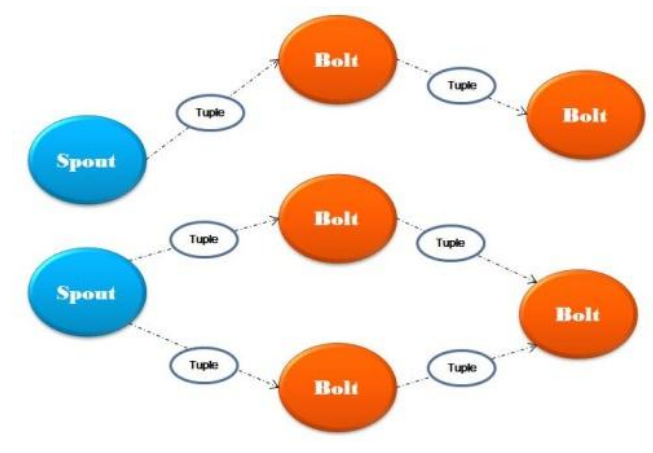

Fig. 2 Conceptual view of Storm

Storm integrates with the queueing and database technologies you already use. There are just three abstractions in Storm: spouts, bolts, and topologies. A spout is a source of streams in a computation. Typically a spout reads from a queueing broker such as Kestrel, RabbitMQ, or Kafka, but a spout can also generate its own stream or read from somewhere like the Twitter streaming API. A bolt processes any number of input streams and produces any number of new output streams. Most of the logic of a computation goes into bolts, such as functions, filters, streaming joins, streaming aggregations, talking to databases, and so on. A topology is a network of spouts and bolts, with each edge in the network representing a bolt subscribing to the output stream of some other spout or bolt. A topology is an arbitrarily complex multi-stage stream computation.

\section{Apache Kafka}

Apache Kafka is publish/subscribe messaging system [15]. Kafka is designed to allow a single cluster to serve as the central data backbone for a large organization. It can be elastically and transparently expanded without downtime. Data streams are partitioned and spread over a cluster of machines to allow data streams larger than the capability of any single machine and to allow clusters of coordinated consumers.

\section{Sentiment Analysis and ML}

Sentiment Analysis techniques can be roughly classified into Lexicon based approach, Machine Learning approach and hybrid approach. The Lexicon-based approach relies on a sentiment lexicon. Lexicon is a collection of known and precompiled sentiment terms. The Machine Learning Approach (ML) applies the famous ML algorithms and it uses linguistic features. The Hybrid Approach combines both approaches and it is very common with sentiment lexicons playing a key role in the majority of methods. We used the hybrid approach to process and analysis our twitter data as it offers the best of two worlds. We are collecting tweets at real-time and these tweets are about all possible topics, including music, fashion, politics, military, economics, trade, health care, movies, current affairs and much more. However, we need to retrieve tweets about politics from this diverse and vast amount of information. Hence, we decided to facilitate some ML algorithm, for this task, namely SVM. In machine learning, support vector machines (SVM) are supervised learning models with associated learning algorithms that analyze data and recognize patterns, used for classification and regression analysis. Given a set of training examples, each marked for belonging to one of two categories, an SVM training algorithm builds a model that assigns new examples into one category or the other, making it a non-probabilistic binary linear classifier. An SVM model is a representation of the examples as points in space, mapped so that the examples of the separate categories are divided by a clear gap that is as wide as possible. New examples are then mapped into that same space and predicted to belong to a category based on which side of the gap they fall on.

\section{E. Description of the Corpus}

We have collected the corpus of public tweets, roughly speaking one-year-data, containing more than 400, 000, 000 tweets so far and still collecting on a daily basis, daily gathering is around $2 \mathrm{mln}$ tweets. Twitter offers two APIs to retrieve data from it: REST API and Streaming API. For this service, we use Streaming API which is a part of Twitter Firehouse and provides $1 \%$ of all tweets. At the first place, it seems little but considering the fact that in every second, on average, around 6,000 tweets are tweeted on Twitter which corresponds to over 350,000 tweets sent per minute, 500 million tweets per day and around 200 billion tweets per year, this $1 \%$ is big enough to perform computation over it.

\section{PROPOSED SYSTEM}

It seems like the world moves at a faster pace every day. People and places become more connected, and people and organizations try to react at an ever-increasing pace. Reaching the limits of human's ability to respond, tools are built to process the vast amounts of data available to decision makers, analyze it, present it, and, in some cases, respond to events as they happen. By their nature, real-time architectures are layered systems that rely on several loosely coupled systems to achieve their goals. There are variety of reasons for this structure, from maintaining high availability of the system in an unreliable world to service requirements and the managing the cost structure of the architecture itself. The remainder of the chapter introduces the overall architecture, workflow and dataflow of the proposed service, SRTAS. Sentiment Analysis is all about extracting opinion from the text. There are various aspects, orientation and reasons of extracting user views in respect to the reason behind the analysis. In our case, it is politics.

\section{A. Workflow of the System}

\section{Preprocessing (data cleaning):}

- Removal of non-English words

- Removal of stop words (a, an, that, the, my ...)

- Case normalization, in this step all incoming tweets are converted into lowercase

2. Feature Selection (Sentiment classification and extraction of political tweets):

- Classification (ML), in this step, all incoming tweets are classified with the help of SVM module.

- Tokenizer is responsible for splitting tweets into meaningful terms or tokens. This procedure can take many types, according to the terminology being used. 
For English, effective tokenization technique is to use white space and punctuation as token delimeters.

- Stemming is the procedure of decreasing relevant tokens into a single type of token. This procedure contains the recognition and elimination of suffixes, prefixes and unsuitable pluralization.

- In Sentiment classification step, we classify the words into positive, negative and neutral lexicons based on the subjectivity of the word. We have to compare each word with predefined word-net libraries. AFINN-111 was used for this task which is a list of English words rated for valence with an integer between minus five (negative) and plus five (positive), with 2477 words and phrases.

\section{Final Phase:}

- Propaganda Labeler, one of the critical parts of our service. In this step, every unique word is counted at real-time and put into dictionary, a list of unique words which is designed to provide word frequency factor and plays a role of influence indication of a word on the context, on a tweet in this case. Under the hood, we used Sliding Window algorithm to keep and demonstrate the word frequency since our service is real-time any word's occurrence is changed all the time.

- DB Writer phase, it is the final step in our system, and after all computation is done the processed tweets are written to the databases, actually two databases. MySQL for real-time view and mongoDB to support long term storage for tweets, so that we can query them in the long run, because MySQL table is used for just real-time, after some period the results in MySQL table are discarded to provide efficiency of real-time view..

Furthermore, there are other steps such as removal of re-tweets and empty tweets, conversion-to ASCII and restoration of abbreviations. We did not mention them in the main part because they are performed after main processing.. You can get more intuitive picture of the system workflow in Pic. 3.

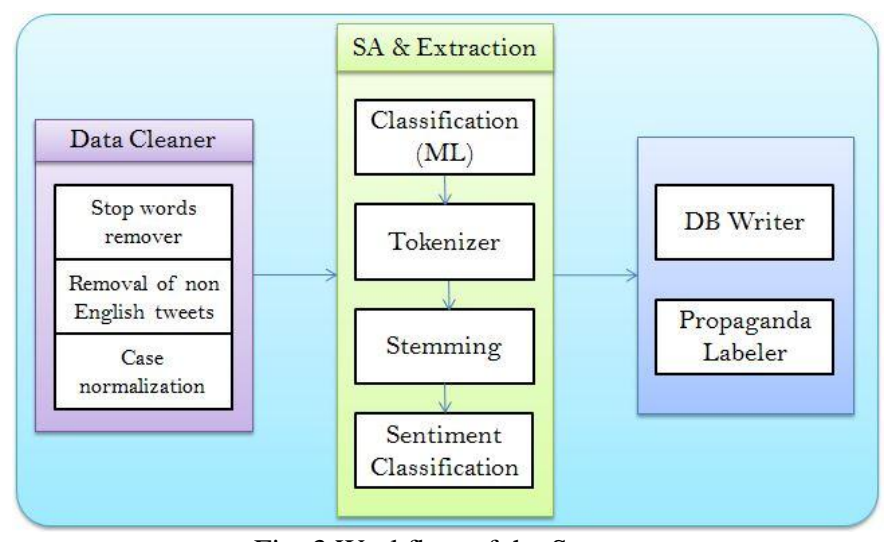

Fig. 3 Workflow of the System

\section{B. Dataflow of the System}

In Pic. 4 the main dataflow of the system is shown. However, first data, streaming of tweets, comes to Kafka for queueing so that we can balance the speed of incoming tweets and maintain dataflow between producer and consumer. Here, producer is twitter crawler which is bound to Twitter's Streaming API for retrieving tweets, and consumer is Storm cluster which is bound to Kafka queue to pull tweets from it. Further, tweets continue their adventure through Storm spout and bolts. After receiving incoming tweets from Kafka topic into Storm spout, they are forwarded to bolts where main business logic is performed. There 4 bolts, and each of them is responsible for certain tasks. First bolt is Tweet- Manipulation Bolt which is responsible for preprocessing phase as before mentioned, all data cleaning is done in this bolt. Then, data flows into Political Aggregator Bolt which shares job, feature selection phase, with Sentiment Analysis Bolt, and both responsible for Sentiment classification and extraction of political tweets. Finally, DB Writer Bolt puts an end to the adventure of the tweets by writing them into databases, MySQL and mongoDB, the former for providing real-time dashboard and the latter for long term storage. You can get more intuitive picture of the system dataflow in Pic. 4 which is given below.

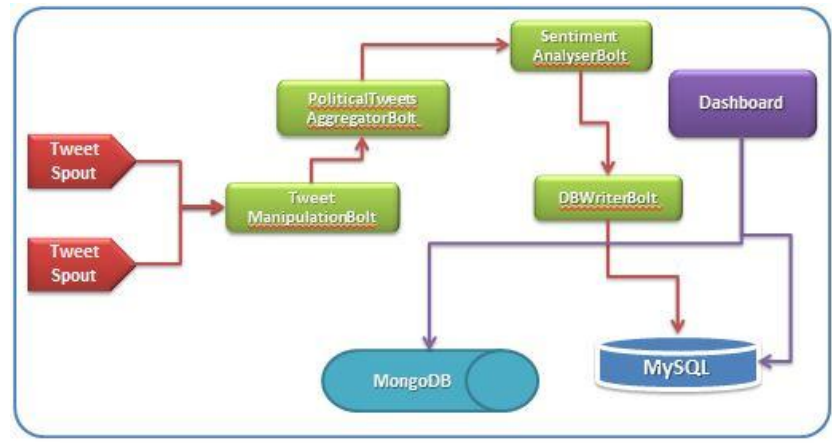

Fig. 4 Dataflow of the System

\section{Identify the Headings}

Finally. we are here to present the architecture of the proposed system. We facilitated a number of Big Data and NoSQL technologies to build our service (SRTAS). Apache Storm, distributed real-time computation system, is at the heart of the system, hence, the main processing unit of the system. Apache Kafka is an ideal source for Storm topologies. It provides everything necessary for:

- At most once processing

- At least once processing

- Exactly once processing

Apache Storm includes Kafka spout implementations for all levels of reliability. Tweets were retrieved in the form of JSON documents. NoSQL document database systems offer convenient functionality to store JSON objects. Therefore, mongoDB database system was chosen as a backend storage for storing tweets. A database in mongoDB is organized in collections, while a collection holds documents. Tweets were stored in different collections.

Dictionary of unique words which is intended to provide propaganda rate and sentiment of these words were later inserted into a relational MySQL database, to allow a presentation of results to users. Further, we used Keen.io to create a real-time dashboard and this dashboard is backed by 
MySQL database where the results of the recent processing is stored. You can get more intuitive picture of the system architecture in Pic. 5 which is given below.

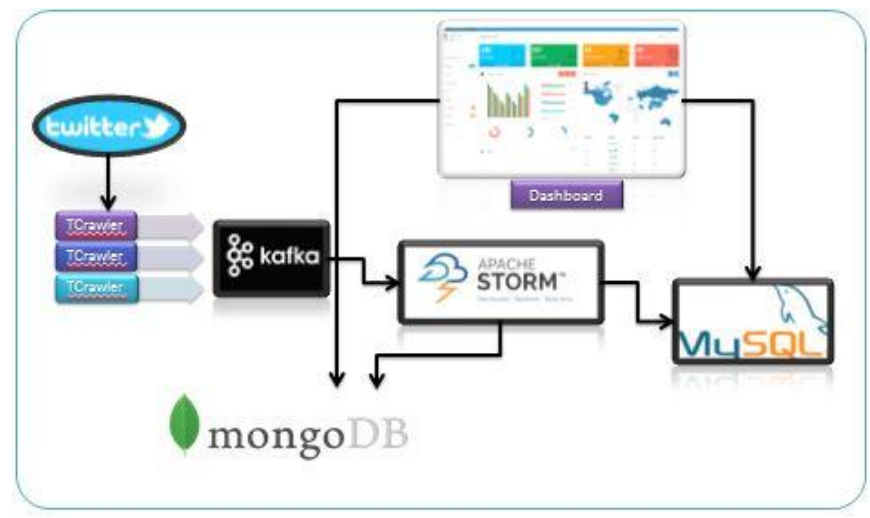

Fig. 5 Architecture of the System

\section{V.CONCLUSION}

Recently, real-time analytics platforms has gained momentum and become integral part of any Big Data architecture of any organization. As variety, velocity and amount of information is increasing at an alarming pace and we need efficient and reliable tools and techniques to tackle the challenge of it. The design and architecture of real-time streaming systems is different from traditional batch oriented systems because we have ever-changing data at our hands which is far more complex and difficult to deal with. In this paper, we present Real Time Analytics Service (SRTAS) which is designed for conducting incremental computation to find propaganda and sentiment analysis of political tweets on cloud computing environment. To build the proposed system we use several cutting-edge techs like Storm, Kafka and mongoDB, moreover, we will leverage well-known ML algorithms and SA techniques efficiently to provide promised service.

\section{ACKNOWLEDGMENT}

This work was supported by Institute for Information \& communication Technology Promotion (IITP) grant funded by the Korea government (MSIP) (R0113-15-0008)

\section{REFERENCES}

[1] Y. R. Lin, J. P. Bagrow, and D. Lazer: "More voices than ever? quantifying media bias in networks." International Conference on Weblogs and Social Media (ICWSM), 2011.

[2] B. Pang and L. Lee. Thumbs up?: sentiment classification using machine learning techniques. Conference on Empirical Methods in Natural Language Processing (EMNLP), 10:79-86, 2002. http://dx.doi.org/10.3115/1118693.1118704

[3] W.H. Lin, T. Wilson, J. Wiebe, and A. Hauptmann. Which side are you on? Identifying perspectives at the document and sentence level. Conference on Natural Language Learning (CoNLL), 2006.

[4] O'Connor, B., Balasubramanyan, R., Routledge, B.R., Smith, N.A.: "From tweets to polls: Linking text sentiment to public opinion time series." In: Proceedings of the International AAAI Conference on Weblogs and Social Media, pp. 122-129 (2010)

[5] Jansen, B.J., Zhang, M., Sobel, K., Chowdury, A.: Micro-blogging as online word of mouth branding. In: Proceedings of the 27th International Conference Extended Abstracts on Human Factors in Computing Systems, pp. 3859-3864 (2009) http://dx.doi.org/10.1145/1520340.1520584

[6] Pak, A., Paroubek, P.: Twitter as a corpus for sentiment analysis and opinion mining. In: Proceedings of the Seventh Conference on International Language Resources and Evaluation, pp. 1320-1326 (2010)

[7] Go, A., Huang, L., Bhayani, R.: Twitter sentiment classification using distant supervision. In: CS224N Project Report, Stanford (2009).

[8] Bifet, A., Frank, E.: Sentiment knowledge discovery in twitter streaming data. In: Pfahringer, B., Holmes, G., Hoffmann, A. (eds.) DS 2010. LNCS, vol. 6332, pp. 1-15. Springer, Heidelberg (2010). http://dx.doi.org/10.1007/978-3-642-16184-1_1

[9] A. Livne, M. Simmons, E. Adar, and L. Adamic: "The party is over here: Structure and content in the 2010 election." International Conference on Weblogs and Social Media (ICWSM), 2011.

[10] M. Wagner. Obama election ushering in first internet presidency. http://www.informationweek.com/news/government/ 212000815, 2008.

[11] Kishori K. Pawar, Pukhraj P Shrishrimal, R. R. Deshmukh: "Twitter Sentiment Analysis: A Review". International Journal of Scientific \& Engineering Research, Volume 6, Issue 4, April-2015

[12] E. Mustafaraj, S. Finn, C. Whitlock, and P. T. Metaxas. Vocal minority versus silent majority: Discovering the opinions of the long tail. International Conference on Social Computing, 2011. http://dx.doi.org/10.1109/passat/socialcom.2011.188

[13] Yelena Mejova, Padmini Srinivasan, Bob Boynton: "GOP Primary Season on Twitter: Popular Political Sentiment in Social Media". WSDM'13, February 4-8, 2012, Rome, Italy.

[14] Storm : https://storm.apache.org/

[15] Kafka : http://kafka.apache.org/ 